\title{
Dopamine and hippocampal input to the nucleus accumbens play an essential role in the search for food in an unpredictable environment
}

\author{
STAN B. FLORESCO and ANTHONY G. PHILLIPS \\ University of British Columbia, Vancouver, British Columbia, Canada
}

\begin{abstract}
The role of $\mathrm{D}_{1}$ and $\mathrm{D}_{2}$ receptors in the nucleus accumbens, and their interaction with hippocampal afferents in mediating exploration, was assessed during a foraging task on a radial-arm maze. In Experiment 1, bilateral infusions into the nucleus accumbens of the $D_{1}$ antagonist $S C H 23390(50,100$, or $500 \mathrm{ng}$ ), but not the $\mathrm{D}_{2}$ antagonist sulpiride (125, 250, or $500 \mathrm{ng}$ ), disrupted foraging for four pellets placed at random on an eight-arm radial maze. In Experiment 2, we examined the effects on foraging of blockade of dopamine receptors in the nucleus accumbens in combination with temporary disruption of information relayed by hippocampal afferents to the ventral striatum. Specifically, we employed an asymmetrical infusion procedure that consisted of a unilateral infusion of SCH 23390 (500 ng) into the nucleus accumbens in combination with a contralateral lidocaine-induced inactivation of the ventral $\mathrm{CA1} /$ subiculum, and this asymmetrical infusion procedure disrupted choice of unexplored arms on the radial-arm maze. These data suggest that mesoaccumbens dopamine transmission selectively modulates hippocampal inputs to the nucleus accumbens via an action at $D_{1}$ receptors when an animal is searching for food in the absence of stimuli that predict where food is located in a complex environment.
\end{abstract}

Exploratory locomotion guided by spatial cues is mediated in part by the hippocampal formation. Lesions to the ventral CA1/subiculum (vSub), the main output region of the hippocampus, reduce exploratory activity in a novel environment (Burns, Annett, Kelley, Everitt, \& Robbins, 1996), and lesions of the dorsal hippocampus impair the recognition of novel spatial configurations in an open field (Save, Poucet, Foreman, \& Buhot, 1992). Chemical stimulation of either the dentate gyrus or the vSub in rats results in an increase in exploratory activity (Mogenson \& Nielson, 1984; Wu \& Brudzynski, 1995; Yang \& Mogenson, 1986). The pattern of exploratory behavior displayed following NMDA infusions into the vSub is qualitatively different from the pattern of locomotor activity observed following systemic administration of psychostimulants (Brudzynski \& Krol, 1997). Moreover, neurons in the dorsal hippocampus or the vSub recorded from freely moving rats exhibit place- and movement-correlated activity when an animal navigates through a spatial environment (Barnes, McNaughton, Mizumori, Leonard, \& Lin, 1990).

Foraging for food on a radial arm maze is one specific type of exploratory locomotion that is dependent on the

This work was supported by a grant from the Natural Sciences and Engineering Research Council of Canada. S.B.F. is a recipient of an NSERC scholarship. We thank Deanna Braaksma and Mark Deeby for their assistance with behavioral testing, Peter Pajor for his computer assistance, and Jeremy Seamans for helpful discussions. Correspondence regarding this article should be addressed to A. G. Phillips, Department of Psychology, University of British Columbia, 2136 West Mall, Vancouver, BC V6T 1Z4, Canada (e-mail: stanbf@unixg.ubc.ca). hippocampus (McDonald \& White, 1993; Olton \& Papas, 1979). We have shown recently that searching for food pellets placed at random on four of eight arms of a radial maze was disrupted following disconnections between the hippocampus and ventral striatum, produced by unilateral lidocaine-induced inactivation of the vSub in one hemisphere, in combination with a contralateral inactivation of the nucleus accumbens (N.Acc.) in the opposite hemisphere (Floresco, Seamans, \& Phillips, 1997). Because rats have no information about the location of food in specific arms on the radial-arm maze, they must first make choices randomly, and as the trial continues, they must avoid arms entered previously, whether or not these arms contained food, and approach novel arms yet to be entered on that trial. The results suggest that spatial information processed by the hippocampus is relayed to the N.Acc. via the vSub and is essential to guide the choice of novel spatial locations during search for food in an environment devoid of specific information about the location of reward (i.e., an unpredictable environment).

Psychopharmacological studies of the neurochemical basis of exploratory locomotion have shown that this behavior also depends on dopamine (DA) neurotransmission in the N.Acc. Increased exploratory activity following NMDA stimulation of the vSub is abolished following DA-depleting lesions of the N.Acc. (Wu \& Brudzynski, 1995). Infusions of DA agonists into the N.Acc. increase locomotor activity (Mogenson, Brudzynski, Wu, Yang, \& Yim, 1993), whereas infusions of the $D A D_{2} / D_{1}$ antagonist fluphenazine selectively decrease locomotor activity, but only in novel environments (Hooks \& Kalivas, 1995). We have observed that infusions of the $D_{2} / D_{1}$ an- 


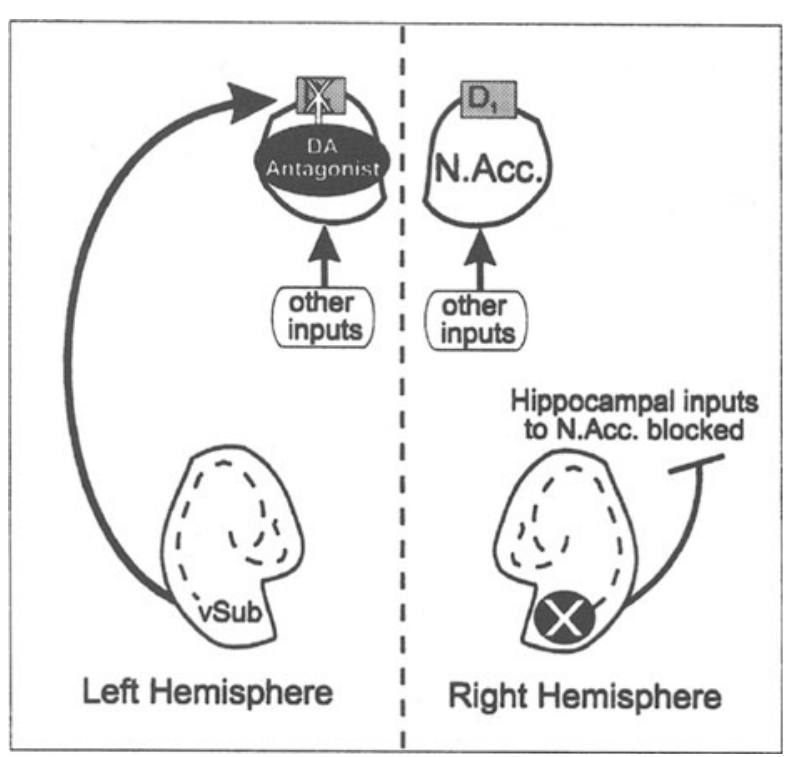

Figure 1. Diagram representing the procedure for assessing dopamine (DA) receptor modulation of hippocampal inputs to the nucleus accumbens (N.Acc.), consisting of a unilateral infusion of a $D_{1}$ receptor antagonist into the N.Acc. and a contralateral infusion of lidocaine into the ventral $\mathrm{CA} 1 /$ subiculum $(\mathrm{X})$. Following this asymmetrical infusion procedure, no inputs would be modulated by $D_{1}$ receptor activity in the left $N$.Acc. Conversely, in the right $\mathbf{N}$.Acc. all inputs except hippocampal afferents would be modulated by DA. Thus, this procedure would prevent DA modulation of hippocampal inputs to the N.Acc. in both hemispheres.

tagonist haloperidol into the N.Acc. selectively disrupt performance during the random foraging radial-arm maze task but have no effect on foraging during a delayed version of this task, when rats have learned about the probable location of food (Floresco, Seamans, \& Phillips, 1996). Thus, DA transmission in the N.Acc. appears to be essential for foraging behaviors dependent on a functional circuit linking the hippocampus and the ventral striatum. In contrast, successful performance of the delayed version of the radial-arm maze has been shown to depend on intact serial transmission between the hippocampus and the prefrontal cortex (PFC; Floresco, Seamans, \& Phillips, 1997).

Anatomical and electrophysiological studies provide detailed information about interactions between hippocampal afferents and mesolimbic DA inputs to the N.Acc. Glutamatergic projections connect the vSub to the medial shell region of the N.Acc. (Brog, Salyapongse, Deutch, \& Zahm, 1993; Fuller, Russchen, \& Price, 1987; Groenewegen, Vermeulen-Van der Zee, te Kortschot, \& Witter, 1987). These projections, in close apposition to DA varicosities (Sesack \& Pickel, 1990; Totterdell \& Smith, 1989), provide a neuroanatomical substrate for DA modulation of hippocampal inputs to the N.Acc. The physiological interactions between hippocampal glutamatergic and mesolimbic DA inputs in the N.Acc. are complex. In earlier in vivo studies, it was reported that iontophoretic application of DA attenuated excitatory responses evoked in N.Acc. neurons following vSub stimulation via a presynaptic action on glutamatergic terminals (Yang \& Mogenson, 1984, 1986, 1987). However, other studies have shown that DA, acting via the $\mathrm{D}_{1}$ receptor, may have no effect or even exert a facilitatory influence on hippocampal inputs to the N.Acc. if these afferents are stimulated at frequencies higher than $1 \mathrm{~Hz}$ (DeFrance, Sikes, \& Chronister, 1985; Gonon \& Sundstrom, 1996; Pennartz, Dolleman-vender Weel, Kitai, \& Lopes da Silva, 1991). These electrophysiological studies suggest that under appropriate conditions, N.Acc. DA transmission may serve to enhance the "signal-to-noise" ratio of information relayed from the hippocampus by selectively augmenting vSub inputs to the N.Acc.

In the present study, we tested two hypotheses, the first being that DA activity in the N.Acc. can influence efficient search patterns for food in a complex environment via the $D_{1}$ receptor subtype. As we have noted above, infusions of the $D_{2} / D_{1}$ receptor antagonist haloperidol into the N.Acc. disrupt the choice of novel arms during the random foraging task (Floresco et al., 1996). Given the recent electrophysiological evidence that $D_{1}$ rather than $\mathrm{D}_{2}$ receptors may play a more important role in the modulation of hippocampal inputs to the N.Acc., it is likely that our results with haloperidol were due to its actions on $D_{1}$ receptors. In Experiment 1, we examined the effects of bilateral infusions of the selective $\mathrm{D}_{1}$ antagonist $\mathrm{SCH}$ 23390 or the selective $D_{2}$ antagonist sulpiride prior to a daily trial of the random foraging task. We also tested a second hypothesis: that the mesolimbic DA projection to the N.Acc. via an action at $D_{1}$ receptors, selectively modulates hippocampal inputs to the N.Acc. during search for food on the random foraging task. In so doing, we utilized a modified disconnection procedure consisting of a unilateral lidocaine-induced inactivation of the vSub in combination with the infusion of $a D_{1}$ antagonist into the contralateral N.Acc. (see Figure 1). In our rationale for this procedure, we assumed (1) that a unilateral infusion of the DA antagonist would ensure that none of the afferents to the N.Acc. in that hemisphere (including those from the vSub) would be modulated by DA; (2) that in the contralateral hemisphere in which the vSub is inactivated, all afferents except those from the hippocampus would be modulated by $\mathrm{DA}$. The $\mathrm{D}_{1}$ receptor antagonist SCH 23390 was infused unilaterally into the N.Acc. at a dose that was effective in disrupting foraging behavior when administered bilaterally. Accordingly, following this asymmetric infusion procedure, there would be no dopaminergic modulation of hippocampal inputs to the N.Acc. in either hemisphere. We had used this procedure previously to assess dopaminergic modulation of hippocampal inputs to the PFC during a delayed variant of the radial-arm maze task, in which rats had previous information about the location of food (Seamans, Floresco, \& Phillips, 1998). 


\section{METHOD}

\section{Subjects and Surgery}

The subjects were male Long Evans rats weighing $300-450 \mathrm{~g}$ prior to surgery. All rats were maintained at $85 \%$ of their free-feeding weights by providing $25-30 \mathrm{~g}$ of Purina lab chow pellets once daily. The rats were tested 5 to 7 days a week, between 1000 and $1400 \mathrm{~h}$ during the light phase of their 12:12-h light:dark cycle.

The rats were anaesthetized with $100 \mathrm{mg} / \mathrm{kg}$ of ketamine hydrochloride and $7 \mathrm{mg} / \mathrm{kg}$ xylazine. Twenty-three-gauge stainless steel guide cannulae were implanted into the brain regions of interest, using standard stereotaxic techniques. The stereotaxic co-ordinates (flat skull) were derived from Paxinos and Watson (1997). For the bilateral injections of $D_{1}$ or $D_{2}$ antagonists, one set of cannulae were implanted bilaterally into the medial N.Acc.: $\mathrm{AP}=+1.4 \mathrm{~mm}$ from bregma, $\mathrm{ML}= \pm 1.3 \mathrm{~mm}$ from midline, and $\mathrm{DV}=-6.1 \mathrm{~mm}$ from dura. The rats in the asymmetrical infusion experiment were implanted with two sets of bilateral cannulae, one pair into the N.Acc. and a second pair into the vSub region of the hippocampus: $A P=$ $-6.0 \mathrm{~mm}$ from bregma, $\mathrm{ML}= \pm 5.5 \mathrm{~mm}$ from midline, and $\mathrm{DV}=$ $-5.3 \pm 0.3 \mathrm{~mm}$ from dura. Thirty-gauge obdurators, flush with the end of the guide cannulae, remained in place until the injections were made. Each rat was given at least 7 days to recover from surgery prior to testing.

\section{Apparatus}

An eight-arm radial maze was used for all experiments. The maze had an octagonal center platform $40 \mathrm{~cm}$ in diameter connected to eight, equally spaced arms, each measuring $50 \times 9 \mathrm{~cm}$, with a cylindrical food cup at the end. The maze was elevated $40 \mathrm{~cm}$ from the floor and was surrounded by numerous extramaze cues (i.e., cupboards, posters, doors, the experimenter, etc.), in a room $4 \times$ $5 \times 3 \mathrm{~m}$, which was illuminated with overhead fluorescent lights $(100 \mathrm{~W})$.

\section{Drugs and Microinjection Protocol}

The selective $D_{1}$ receptor antagonist SCH $23390(50,100$, or $500 \mathrm{ng} / 0.5 \mu \mathrm{l}$; Research Biochemicals, Natick, MA) was dissolved in physiological saline. The selective $\mathrm{D}_{2}$ receptor antagonist sulpiride $(125,250$, or $500 \mathrm{ng} / 0.5 \mu \mathrm{l}$; Research Biochemicals) was dissolved in a drop of $\mathrm{NaOH}$ and phosphate-buffered saline.

On injection days, the obdurators were removed and 30-gauge stainless steel injection cannulae were inserted $0.8 \mathrm{~mm}$ beyond the tip of the guide cannulae. In Experiment 1, bilateral infusions of SCH 23390 , sulpiride, or vehicle were delivered into the N.Acc. at a rate of $0.5 \mu \mathrm{l} / 52 \mathrm{sec}$ by a microsyringe pump (Sage Instruments, Model 341). Injection cannulae were left in place for an additional $1 \mathrm{~min}$ to allow for diffusion. Each rat remained in its home cage for a further $7 \mathrm{~min}$ period prior to behavioral testing.

In Experiment 2, the rats received a unilateral infusion of $\mathrm{SCH}$ $23390(500 \mathrm{ng})$ or vehicle into the N.Acc., according to the same protocol as in Experiment 1. This was followed by an infusion of either lidocaine ( $20 \mu \mathrm{g}$ in $0.5 \mu \mathrm{l}$ of saline; Astra Pharmaceuticals, Mississuaga, $\mathrm{ON}$, and Research Biochemicals) or vehicle into the $v$ Sub, delivered at a rate of $0.5 \mu \mathrm{l} / 1.2 \mathrm{~min}$. Injection cannulae were left in place for an additional $1 \mathrm{~min}$ following each injection to allow for diffusion. Each rat remained in its home cage for an additional $3.5 \mathrm{~min}$ prior to behavioral testing.

\section{Behavioral Procedure}

The random foraging task has been described elsewhere (Floresco et al., 1996; Floresco, Seamans, \& Phillips, 1997). On the first 2 days of testing, the rats were habituated to the maze environment. Subsequent training trials were given once daily. On these daily trials, the animals were required to forage for pellets placed at random in the food cups of four of the eight arms. A novel set of arms was baited each day. Optimal foraging strategy required the rat to enter novel arms not visited previously during the daily trial and to avoid the arms that had been entered. The animals were removed from the maze once they had retrieved all four pellets or $10 \mathrm{~min}$ had elapsed. They were trained to a criterion of no more than one reentry error per daily trial for 4 consecutive days. The day after criterion performance had been achieved, the first intracranial injections were administered. On these injection test days, the rats were removed from the maze only after all four pellets had been retrieved. Following the 1 st injection day, the animals were retrained to criterion for 2 consecutive days. This procedure was repeated until each animal had received all the designated injections.

Errors were scored as reentry into arms visited previously within a trial. These errors were broken down further into reentry into baited arms (arms that had been baited at the start of the trial) and reentry to nonbaited arms (arms that were not baited prior to the start of the trial). The numbers of reentry errors made on each of the injection days were recorded and used for data analysis. The rats' latencies to reach the first food cup (either baited or nonbaited) after placement on the maze and the time that they required in order to retrieve all four pellets were also recorded.

\section{Design and Procedure}

Experiment 1: Bilateral infusions of SCH 23390 or Sulpiride into the N.Acc. A within-subjects design was used with both drugs in Experiment 1 . Two groups of rats with bilateral cannulae implanted into the N.Acc. were trained on the random foraging task. On the 1st injection day, each group of rats was assigned to two subgroups, one of which received bilateral infusions of either SCH $23390(50,100$, or $500 \mathrm{ng})$ or vehicle, whereas the other group received sulpiride $(125,250$, or $500 \mathrm{ng})$ or vehicle into the N.Acc. prior to a daily random foraging trial. The animals were subsequently retrained until they reattained criterion performance. On the day after criterion had been reattained, a second infusion of either drug or vehicle was administered in a counterbalanced order. This procedure was repeated until each rat had received all three drug doses and vehicle. The order of injections was counterbalanced between animals, using a quasi-Latin square design. The counterbalancing was designed to ensure that a given sequence of injections was not repeated.

Experiment 2: Asymmetrical infusions of lidocaine into the vSub and a $D_{1}$ antagonist into the N.Acc. A within-subjects design was used for Experiment 2. One group of rats with two pairs of cannulae implanted bilaterally into both the vSub and the N.Acc. was trained on the random foraging task. This group received unilateral infusions of SCH 23390 into the N.Acc. in combination with lidocaine in the contralateral vSub.

The animals in the asymmetrical infusion experiment received a total of four injection procedures prior to daily trials on the random foraging task. The following combinations of asymmetrical bilateral infusions were employed: (1) a unilateral lidocaine inactivation of the vSub in combination with a contralateral infusion of $\mathrm{SCH}$ 23390 (500 ng) into the N.Acc.; (2) a unilateral inactivation of the vSub in combination with a vehicle injection into the contralateral N.Acc.; (3) a unilateral infusion of SCH 23390 into the N.Acc. in combination with a saline injection into the contralateral vSub; and (4) unilateral injections of saline into the vSub and saline injections into the contralateral N.Acc. The order of injections was counterbalanced between animals, using a quasi-Latin square design to ensure that a given sequence of injections was not repeated. The hemisphere (left or right) used for the 1 st injection was also counterbalanced and was alternated for subsequent injections. The injection procedure was repeated until an animal had been tested four times with each sequence of intracranial injections.

\section{Histology}

Upon completion of behavioral testing, the rats were sacrificed in a carbon dioxide chamber. Their brains were removed and fixed 
in a $10 \%$ formalin solution. The brains were frozen and sliced in $50-$ $\mu \mathrm{m}$ sections prior to being mounted and stained with cresyl violet. Placements were verified with reference to the neuroanatomical atlas of Paxinos and Watson (1997).

\section{Data Analysis}

The number and type of errors made on the day prior to the $1 \mathrm{st}$ injection sequence and on all injection days in each experiment were analyzed in separate two-way, repeated measures analyses of variance (ANOVAs), with treatment day and error type as withinsubjects factors. Main effects of treatment were further analyzed, using Tukey's post hoc tests for repeated measures. A significant main effect of treatment was found for Experiment 2. One planned comparison was then made, in order to analyze the number of each type of error made on trials following vehicle injections and on trials in which lidocaine in the vSub was combined with SCH 23390 in the contralateral N.Acc.

When a significant main effect of treatment was found for Experiment 2, three one-way ANOVAs were conducted, to assess the number of errors made on tests after a unilateral inactivation of the vSub or unilateral infusions of SCH 23390 into the N.Acc., and after infusions of lidocaine and SCH 23390 into the vSub and N.Acc. respectively. The side of the injection was the between-subjects factor. This analysis was conducted to rule out the possibility that unilateral inactivations or infusions of $\mathrm{SCH} 23390$ in one hemisphere would lead to a greater increase in errors than would inactivations of the other hemisphere.

The latency data to reach the first food cup and the average time per subsequent choice on the day prior to the 1st injection sequence and on all injection days in each experiment were analyzed with separate one-way repeated measures ANOVAs. Because of the high variance on these latency measures both between and within subjects, the analyses of these data were conducted on the square root transform of the raw latency scores. The average time per subsequent choice was calculated according to the formula:

(Time to complete trial) - (Time to initiate trial)

(Number of choices for trial) -1

\section{RESULTS}

\section{Experiment 1}

SCH 23390. On four separate daily trials, 9 rats received bilateral infusions of SCH $23390(50,100$, or $500 \mathrm{ng}$ ) or vehicle into the N.Acc. prior to a random foraging test trial. Analysis of the number of errors revealed a significant main effect of treatment $[F(4,32)=6.68$, $p<.001$; Figure 2A]. Tukey's post hoc analysis showed that rats made significantly more errors following infusions of the 500-ng dose of SCH 23390 versus all other treatments $(p<.001$, vs. saline and day prior to the 1 st injection; $p<.05$, vs. $100 \mathrm{ng}$ and $50 \mathrm{ng}$ ). Rats made significantly more errors following infusion of either the 100 - or the 50-ng dose relative to the day prior to the 1 st injection $(p<.05)$. No other treatment conditions differed from each other. In addition, there were no significant main effects of error type or treatment $\times$ error type interactions (all $F \mathrm{~s}<2.1$, n.s.), indicating that the rats in all treatment conditions made an equivalent number of reentries to both baited and nonbaited arms.

Analysis of the latency data revealed that infusions of SCH 23390 did not affect the latency to initiate responding $[F(4,32)=0.86, \mathbf{n} . \mathbf{s}$.]. However, a separate analysis showed that infusions of the 500-ng dose of SCH 23390 significantly increased the average time per subsequent choice relative to saline treatment or the day prior to the 1 st injection $[F(4,32)=3.52, p<.05$, and Tukey's, $p<$ $.05]$. There were no other differences between any treatment conditions on this latency measure. The latency data for rats receiving infusions of $\mathrm{SCH} 23390$ into the N.Acc. are presented in Table 1A.

Sulpiride. A separate group of 7 rats received bilateral infusions of sulpiride $(125,250$, or $500 \mathrm{ng})$ or vehicle into the N.Acc. prior to four random foraging trials. Although infusions of the 500-ng dose slightly increased the number of errors made by the rats during the random foraging task, the overall analysis showed no significant difference between treatments $[F(4,24)=1.28$, n.s.; Figure $2 \mathrm{~B}$ ]. The increase in the mean number of errors made by the rats following infusions of the 500-ng dose of sulpiride were due mainly to 2 rats that showed notable disruption in foraging behavior ( $M$ errors $=6.0$ ). However the remaining 5 rats in this group did not exhibit any major disruption in foraging accuracy following this treatment $(M$ errors $=1.0)$. Furthermore, there were no significant main effects of error type or treatment $\times$ error type interactions (all $F \mathrm{~s}<1.75$, n.s.).

Analysis of the latency data revealed that infusions of sulpiride had no significant effect on latencies to initiate foraging relative to vehicle infusions $[F(4,24)=1.21$, n.s.]. Infusions of 500-ng dose of sulpiride enhanced the average time per subsequent choice relative to saline treatment or the day prior to the lst injection $[F(4,32)=3.07$, $p<.05$, and Tukey's, $p<.05]$. There were no other differences between any treatment conditions on this latency measure. The latency data for rats receiving infusions of sulpiride into the N.Acc. are presented in Table 1A.

Histology. The location of the cannulae tips for all animals in Experiment 1 are presented in Figure 2C. All the animals had infusions that were located mainly or entirely in the medial shell region of the N.Acc.

\section{Experiment 2}

Effects of asymmetrical inactivation of the vSub in combination with $\mathrm{SCH} 23390$ injections into the $\mathrm{N}$.Acc. Analysis of the number of errors made by the rats $(n=8)$ following asymmetrical infusion of lidocaine and $\mathrm{SCH}$ 23390 into the vSub and N.Acc., respectively, or control treatments revealed a significant main effect of treatment $[F(4,28)=3.16, p<.05]$. Tukey's post hoc test for repeated measures confirmed that the rats made significantly more errors following unilateral inactivations of the vSub in combination with contralateral infusions of $\mathrm{SCH}$ 23390 into the N.Acc. versus all other injection protocols $(p<.01)$, and that control injection protocols did not differ significantly from each other (see Figure 3A). Subsequent planned comparisons of the type of errors made after experimental and control treatments revealed no significant effect of treatment on the number of reentries into baited versus nonbaited arms $[F(1,7)=1.36$, n.s.; see Figure $3 \mathrm{~A}$, inset]. There were no significant main effects of 


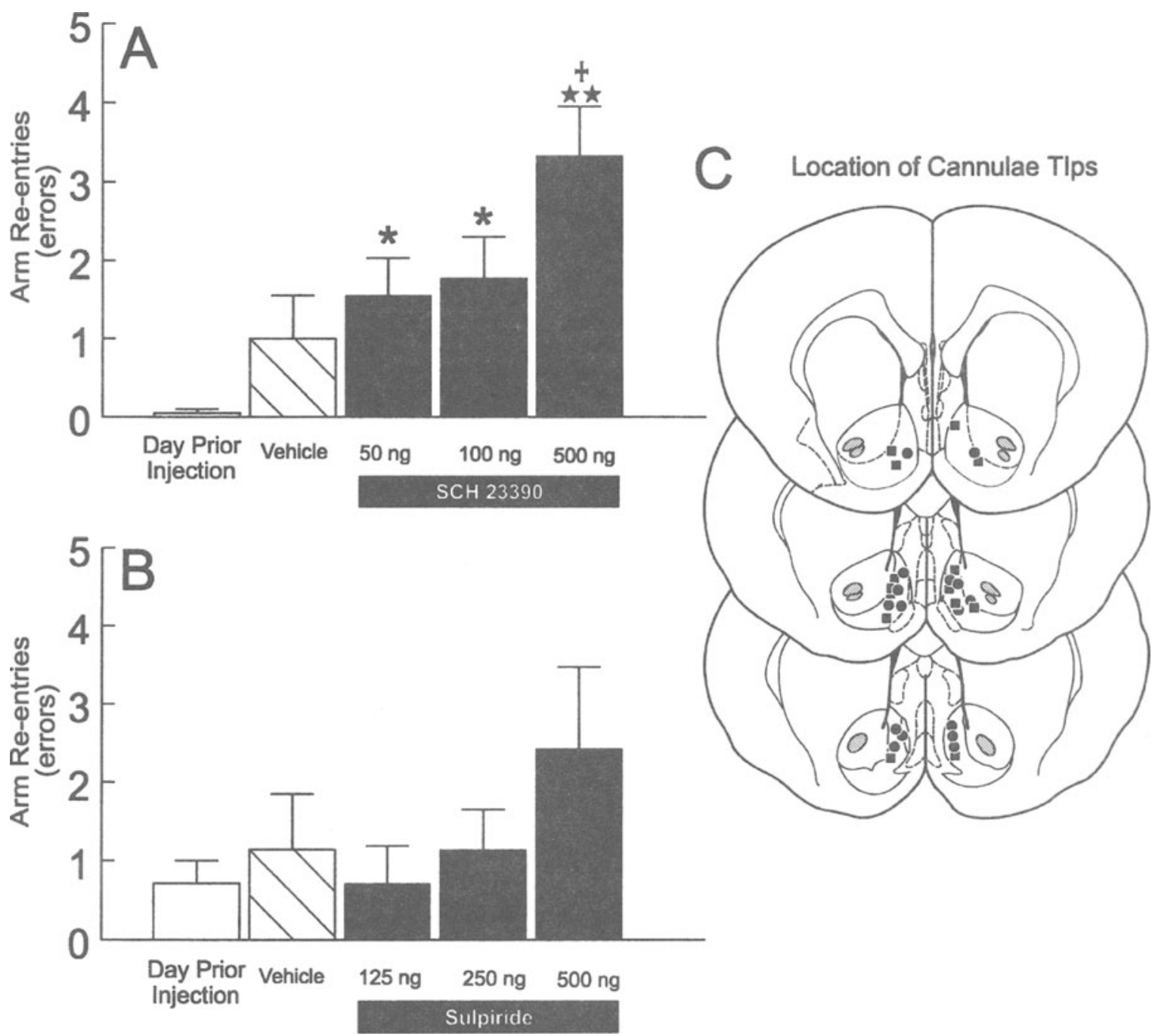

Figure 2. The effects of bilateral injections of a $D_{1}$ (SCH 23390) or $D_{2}$ (sulpiride) antagonist into the nucleus accumbens (N.Acc.) of rats well trained on the random foraging task. (A) Number of errors $(M \pm S E M)$ made during the random foraging task on the days prior to the 1 st injection (open bar) and following injections of saline (hatched bar), 50, 100, or $500 \mathrm{ng}$ of SCH 23390 (black bars) into the N.Acc. Double stars denote significance at $p<.001$ relative to saline injections and to the day prior to the 1 st injection. Cross denotes significance at $p<.05$ relative to 100 -ng and 50-ng doses. The asterisk denotes significance at $p<.05$ relative to the day prior to the first injection. (B) Number of errors $(M \pm S E M)$ made during the random foraging task on the day prior to the 1st injection (open bar) and following injections of phosphate-buffered saline (hatched bar), 125, 250, or 500 ng of sulpiride (black bars) into the $N$.Acc. (C) Schematic representation showing location of cannulae tips for all rats receiving infusions of SCH 23390 (black dots) and sulpiride (black squares) into the N.Acc. in Experiment 1. Only eight dots are shown due to overlap of placement. Plates are adaptations from Paxinos and Watson (1997).

error type or treatment $\times$ error interactions (all $F \mathrm{~s}<1.1$, n.s.). Similarly, there were no hemispheric biases in the number of errors made following unilateral vSub inactivations, unilateral N.Acc. infusions of SCH 23390 , or lidocaine-vSub/SCH 23390-N.Acc. injections (all Fs < 0.50, n.s.). The rats that received asymmetrical infusions with SCH 23390 made more errors than did the rats that received infusions of the 500-ng dose of SCH 23390 bilaterally, but this difference was not significant $[F(1,15)=$ 0.87, n.s.]. Finally, there were no significant differences between treatment conditions on the latencies to reach the first food cup or the average time per subsequent choice (all $F$ s $<2.1$, n.s.). The latency data for rats receiving asymmetrical infusions of SCH 23390 into the N.Acc. and lidocaine into the vSub are presented in Table 1B.

Histology. The location of the cannulae tips for all animals receiving N.Acc.-vSub asymmetrical infusions are represented in Figure 3B. Bilateral placements in the vSub were similar to those observed previously (Floresco, Seamans, \& Phillips, 1997). Similarly, bilateral placements in the N.Acc. were within the same regions of the ventral striatum as those observed in Experiment 1. 
Table 1A

Latency Data (in Seconds) for Experiment 1

\begin{tabular}{|c|c|c|c|c|c|c|c|c|c|c|c|c|c|c|c|c|}
\hline \multirow[b]{3}{*}{ Latency } & \multirow{2}{*}{\multicolumn{2}{|c|}{ Day Prior }} & \multirow{2}{*}{\multicolumn{2}{|c|}{ Vehicle }} & \multicolumn{6}{|c|}{ SCH 23390} & \multicolumn{6}{|c|}{ Sulpiride } \\
\hline & & & & & \multicolumn{2}{|c|}{$50 \mathrm{ng}$} & \multicolumn{2}{|c|}{$100 \mathrm{ng}$} & \multicolumn{2}{|c|}{$500 \mathrm{ng}$} & \multicolumn{2}{|c|}{$125 \mathrm{ng}$} & \multicolumn{2}{|c|}{$250 \mathrm{ng}$} & \multicolumn{2}{|c|}{$500 \mathrm{ng}$} \\
\hline & $M$ & $S E M$ & $M$ & $S E M$ & $M$ & $S E M$ & $M$ & $S E M$ & $M$ & $S E M$ & $M$ & $S E M$ & $M$ & $S E M$ & $M$ & $S E M$ \\
\hline Time to initiate & 47.1 & 8.1 & 37.0 & 11.9 & 37.6 & 7.8 & 48.5 & 7.5 & 43.8 & 18.5 & & & & & & \\
\hline $\begin{array}{l}\text { Avg. time to } \\
\text { subsequent choice }\end{array}$ & 21.9 & 2.1 & 21.9 & 6.0 & 36.4 & 9.8 & 37.9 & 6.5 & 46.7 & $10.9^{*}$ & & & & & & \\
\hline $\begin{array}{l}\text { Time to initiate } \\
\text { Avg. time to }\end{array}$ & 72.4 & 17.1 & 42.9 & 10.4 & & & & & & & 43.1 & 11.2 & 63.3 & 26.1 & 34.3 & 7.7 \\
\hline subsequent choice & 30.5 & 6.7 & 39.3 & 14.7 & & & & & & & 58.5 & 13.1 & 74.6 & 30.1 & 129.9 & $61.9^{*}$ \\
\hline
\end{tabular}

${ }^{*} p<.05$ versus vehicle and day prior to injection.

Note that Figure 3B represents the asymmetric infusion procedure (see legend for details).

\section{DISCUSSION}

The data from Experiment 1 indicate that activation of the $D_{1}$ receptor in the N.Acc. is required for accurate foraging behavior by rats that have no prior knowledge about the location of food. Local infusion of the $\mathrm{D}_{1}$ receptor antagonist SCH 23390 increased the number of errors made during the random foraging task, whereas treatment with of the $\mathrm{D}_{2}$ antagonist sulpiride did not impair the efficiency of foraging behavior. The present results clarify one of our previous findings (Floresco et al., 1996), by suggesting that the impairment in foraging behavior on the random foraging task following infusions of haloperidol was mediated primarily by blockade of $D_{1}$ receptors, with $\mathrm{D}_{2}$ receptors possibly playing a more permissive role. Other psychopharmacological studies have reported that infusion of a $D_{1}$ receptor agonist into the N.Acc. causes robust and consistent increases in locomotor activity, whereas infusion of a $D_{2}$ agonist has variable effects on locomotion (Wu, Brudzynski, \& Mogenson, 1993). Moreover, systemic administration of SCH 23390 has been shown to block novelty-seeking behavior at doses that have no effect on locomotion, whereas injections of sulpiride only disrupt such behavior at doses that attenuate locomotor activity (Bardo et al., 1993).

Infusions of either SCH $23390(500 \mathrm{ng})$ or sulpiride $(500 \mathrm{ng})$ enhanced significantly the average latencies per choice, but had no effect on the latency to initiate responding at the start of the foraging trial. The fact that sulpiride increased response latencies without effecting the number of errors is consistent with reports that $D_{2}$ antagonists can enhance response latencies during different reward-related tasks, while leaving the accuracy of responding intact (Blackburn, Phillips, \& Fibiger, 1987; Cador et al., 1991; Salamone, Cousins, \& Snyder, 1997). It is also noteworthy that the 500-ng dose of sulpiride produced a greater than threefold increase in the average time per subsequent choice relative to vehicle treatment, whereas the same dose of SCH 23390 was less effective. These latency data, coupled with the increase in errors, suggest that $D_{1}$ receptor activity in the N.Acc. is involved in the accuracy of choosing specific arms, whereas $D_{2}$ receptors may be more involved in influencing response latency.

In Experiment 2, blockade of efferent activity in the hippocampus by unilateral infusions of lidocaine into the vSub coupled with infusions of the $\mathrm{D}_{1}$ antagonist $\mathrm{SCH}$ 23390 into the contralateral N.Acc. significantly disrupted efficient foraging during the random foraging task. It is important to note that unilateral infusions of $\mathrm{SCH}$ 23390 into the N.Acc. or of lidocaine into the vSub, had no significant effect on foraging behavior. In a previous study, we used asymmetrical injections of lidocaine into both the vSub and the N.Acc. to determine the essential role of a hippocampal-ventral striatal circuit in efficient exploratory goal-directed locomotion (Floresco, Seamans, \& Phillips, 1997). The logic underlying the use of this disconnection procedure to identify components of a functional neural circuit is based on the assumption that information is transferred serially from one structure to an efferent region, on both sides of the brain in parallel. Furthermore, the procedure assumes that dysfunction will result from blockade of neural activity at the origin of the pathway in one hemisphere and the termination of the efferent pathway in the contralateral hemisphere. The present finding that blockade of DA receptors in the N.Acc. in combination with a reversible lesion of the vSub disrupted an efficient search strategy on the random foraging task is consistent with the hypothesis that DA in the N.Acc. can modulate selectively hippocampal signals to the ventral striatum when an animal is engaged in exploratory locomotion (Mogenson et al., 1993).

The present findings suggest an important role for DA in the N.Acc. in situations where the animal must learn by chance the location of rewarding stimuli in the test environment. When responding is guided by previously acquired information about the location of a goal object or by conditioned reinforcers, performance is unaffected by depletion of DA or infusions of DA antagonists into the N.Acc. (Floresco et al., 1996; Kim \& Levin, 1996; Ploeger, Spruijt, \& Cools, 1994; Robbins \& Everitt, 1992; Wolterink et al., 1993). Parallels may exist between the importance of DA in the N.Acc. for efficient food searching strategies in the radial-arm maze task and its importance in the acquisition of a conditioned place-preference 

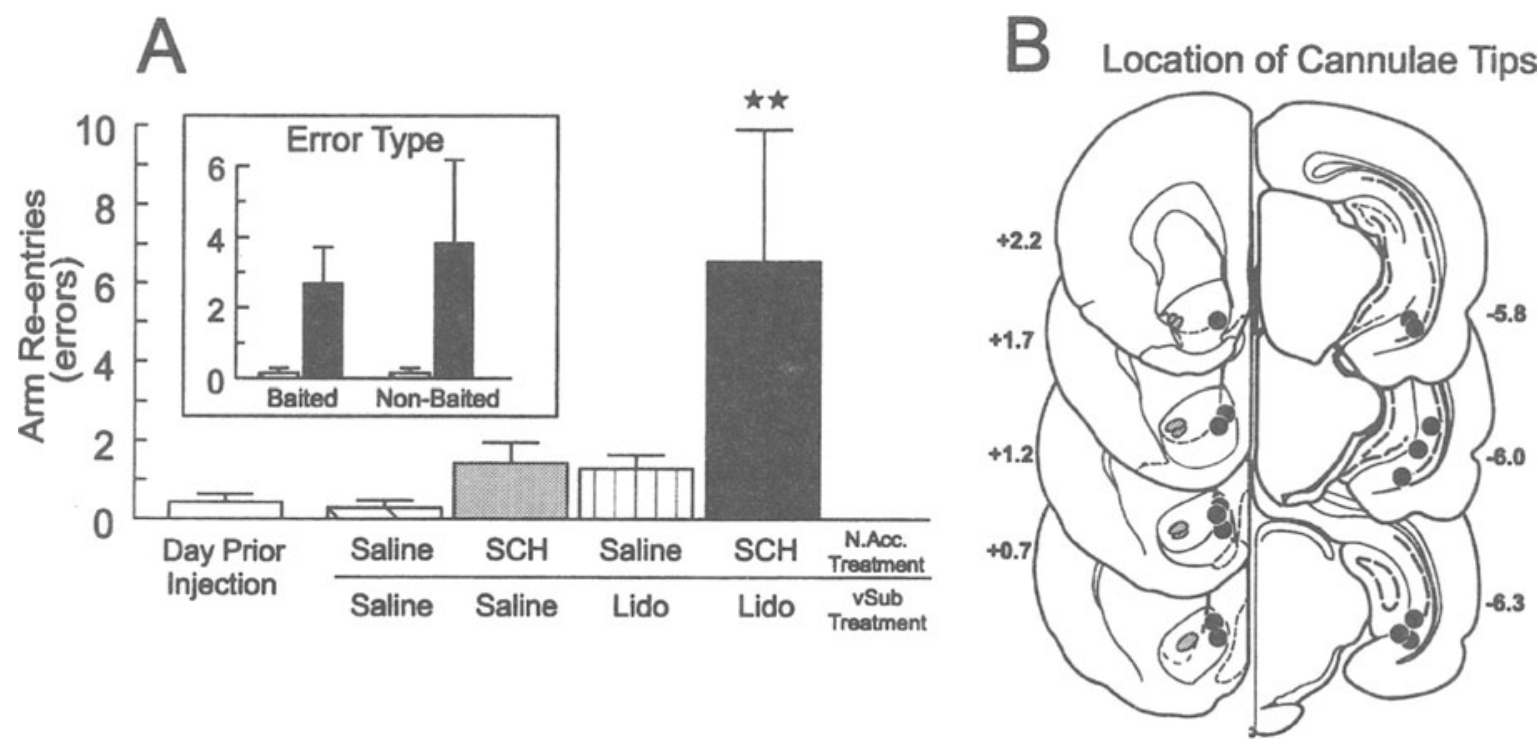

Figure 3. The effect of unilateral inactivation of the ventral CA1/subiculum (vSub) in combination with unilateral injections of SCH 23390 into the nucleus accumbens (N.Acc.) in the contralateral hemisphere. (A) Number of errors $(M \pm S E M)$ made during the random foraging task by rats on the days before the 1st injection (open bar), after unilateral infusions of saline into both the N.Acc. and vSub (hatched bar), unilateral infusions of SCH 23390 (SCH, $500 \mathrm{ng}$ ) into the N.Acc. and contralateral infusions of saline into the vSub (gray bar), unilateral infusions of saline into the $N$.Acc. and contralateral infusions of lidocaine into the vSub (striped bar), and unilateral injections of $\mathrm{SCH}(500 \mathrm{ng})$ into the $\mathrm{N}$.Acc. and contralateral injections of lidocaine into the vSub (black bar). Double stars denote significance at $p<.01$ versus all other treatment conditions. Inset shows number of reentries to baited arms and nonbaited arms on saline/saline (hatched bar) and SCH 23390/Lido (black bar) injection days. (B) Schematic representation of the SCH 23390 injection sites into the N.Acc. and lidocaine injection sites into the vSub for all rats in Experiment 2 . Black dots represent the location of cannula tips. Illustrated brain sections are computergenerated adaptations from Paxinos and Watson (1997). Numbers beside each plate correspond to mm from bregma. For clarity, panel B represents the location of cannulae tips on sides that received infusions on disconnection injection days. All animals received infusions of SCH 23390 , lidocaine, or saline in each hemisphere.

for food (Spyraki, Fibiger, \& Phillips, 1982a) and psychostimulant drugs (Hiroi \& White, 1990; Spyraki, Fibiger, \& Phillips, 1982b). In both situations, the animals must acquire and act on information of a contextual nature that is predictive of reward. In future experiments, it would be most interesting to examine the effects of the asymmetrical infusion procedure employed here on a conventional conditioned place-preference procedure with food or drugs as reward. A disruption of the acquisition of place preference would be consistent with a role for DA in the N.Acc. in modulating contextual information or stimulus-reward associations processed by the hippocampus or the amygdala (Everitt, Morris, O'Brien, \& Robbins, 1991).

\section{Physiological Evidence for DA Modulation of Hippocampal Afferents to the Nucleus Accumbens}

Contemporary theory posits that the transfer of information from the hippocampus to ventral striatum is required in order to direct and guide behavior toward novel or unexpected stimuli (Berns, Cohen, \& Mintun, 1997; Buhusi \& Schmajuk, 1996; Burns et al., 1996; Floresco, Seamans, \& Phillips, 1997), and the present data highlight the essential role of mesoaccumbens DA activity in facilitating signal transmission between these two brain regions during exploration of an unpredictable environment. It is well established that hippocampal or cortical glutamatergic inputs to the N.Acc. arriving at low frequencies $(\leq 1 \mathrm{~Hz})$ are attenuated by DA, presumably by a presynaptic mechanism (Harvey \& Lacey, 1996; Pennartz et al., 1991; Yang \& Mogenson, 1986). In contrast, other studies suggest that DA can facilitate higher frequency hippocampal inputs to the N.Acc. Intracellular recordings from N.Acc. neurons in vitro have shown that application of DA can augment paired-pulse facilitation following stimulation of the fimbria (Pennartz et al., 1991; Pennartz, Groenewegen, \& Lopes da Silva, 1994). Similarly, increased N.Acc. DA transmission caused by NMDA-induced stimulation of the ventral tegmentum can also augment vSub-evoked excitatory responses in the ventral striatum in vivo: an effect abolished by SCH 23390 (Gonon \& Sundstrom, 1996). In the latter study, it was concluded that at lower concentrations, DA in the N.Acc. acts as an excitatory neurotransmitter, a finding that is consistent with other reports that iontophoretic application of low doses of DA or DA agonists in vivo augment excitatory responses in N.Acc. neurons evoked by exogenously applied glutamate (Hu \& Wang, 1988; Kiyatkin \& Rebec, 1996). Taken together, these electrophysiological studies support the hypothesis that DA transmission in the N.Acc. may serve to enhance the "signal-to-noise" 
Table 1B

Latency Data (in Seconds) for Experiment 2

\begin{tabular}{|c|c|c|c|c|c|c|c|c|c|c|}
\hline \multirow[b]{3}{*}{ Latency } & & & & & \multicolumn{6}{|c|}{ Lidocaine vSub-SCH 23390 N.Acc. } \\
\hline & \multicolumn{2}{|c|}{ Day Prior } & \multicolumn{2}{|c|}{ Sal/Sal } & \multicolumn{2}{|c|}{ Sal/Lido } & \multicolumn{2}{|c|}{$\mathrm{SCH} / \mathrm{Sal}$} & \multicolumn{2}{|c|}{ SCH/Lido } \\
\hline & $M$ & $S E M$ & $M$ & $S E M$ & $M$ & $S E M$ & $M$ & $S E M$ & $M$ & $S E M$ \\
\hline Time to initiate & 26.0 & 7.4 & 18.5 & 2.9 & 14.1 & 3.9 & 20.9 & 5.4 & 24.3 & 3.8 \\
\hline $\begin{array}{l}\text { Avg. time to } \\
\text { subsequent choice }\end{array}$ & 15.7 & 1.8 & 9.7 & 1.2 & 11.8 & 1.5 & 12.7 & 2.5 & 15.8 & 1.5 \\
\hline
\end{tabular}

ratio of information from the hippocampus by selectively augmenting vSub inputs to the N.Acc. (DeFrance et al., 1985; Kiyatkin \& Rebec, 1996).

Recordings from the vSub and the N.Acc. in behaving rats provide further insight into the interactions between these two brain regions during exploratory behavior. In addition to well-documented "place-cell" activity (Muller, 1996; O'Keefe \& Nadel, 1978), neurons in both the vSub and N.Acc. exhibit increased high-frequency activity (i.e., $10-30 \mathrm{~Hz}$ ) when a rat initiates a goal-directed response toward the end of an arm on a radial maze (Barnes et al., 1990; Lavoie \& Mizumori, 1994). It has been posited that the movement-correlated activity of N.Acc. neurons under these conditions is driven by inputs from the hippocampus (Lavoie \& Mizumori, 1994). It is therefore reasonable to suggest that when a rat is oriented toward a novel spatial location that has not been approached previously during the trial, high-frequency glutamatergic inputs from the vSub will activate a particular ensemble of N.Acc. neurons (Pennartz et al., 1994), thereby initiating movement toward this novel location. In part on the basis of the present findings and previous electrophysiological studies, it may be conjectured that DA activity in the N.Acc. can augment the effect that higher frequency $(>4 \mathrm{~Hz})$ vSub inputs exert on N.Acc. neurons, thereby increasing the activity in the subset of N.Acc. neurons that govern approach behavior toward novel stimuli. Pennartz and colleagues have proposed a specific mechanism within the N.Acc. by which "short-acting clusters of excitatory rhythmic slow activity input $(4-12 \mathrm{~Hz})$. . may be amplified ... [and] this 'time-domain filtering' appears to be modulated by dopamine" (Pennartz et al., 1994, p. 747).

The deficits in foraging behavior following unilateral inactivation of the vSub and blockade of $D_{1}$ receptors in the contralateral N.Acc. could be attributed to the following sequence of events. Inactivation of the vSub in one hemisphere would deprive the ventral striatum of spatial information that identifies novel arms on the maze. Blockade of DA receptors in the contralateral N.Acc. would disrupt the amplification of hippocampal signals by DA and thereby reduce the activation of the target neuronal ensemble in the N.Acc. As a result of these two manipulations, the activity of $\mathrm{N}$.Acc. neurons normally driven by hippocampal input in both hemispheres would be compromised, resulting in random patterns of locomotion in the maze environment.
One issue that deserves further consideration is the underlying neural mechanism by which N.Acc. DA transmission is increased during exploratory locomotion. Recordings from DA neurons in the ventral tegmental area in awake primates reveal that these cells show selective increases in activity when the animal is presented with novel or unexpected stimuli during a daily training trial (Schultz, 1997), a feature shared with those that a rat would encounter during a daily random foraging trial. Thus, increased activity in the ventral tcgmental area may be partly responsible for enhanced DA transmission during random foraging behavior. However, recent anatomical and neurochemical studies suggest that presynaptic modulation of DA terminals, by activity of direct glutamatergic inputs from the vSub, also may increase DA release in the N.Acc. (Blaha, Yang, Floresco, Barr, \& Phillips, 1997; Gracy \& Pickel, 1996; Totterdell \& Smith, 1989). Therefore, afferent inputs from the vSub may serve a dual function: (1) to activate the particular ensemble of N.Acc. neurons that mediate approach responses to novel stimuli and (2) to increase DA efflux via a presynaptic mechanism that in turn would enhance the influence of hippocampal input on neuronal activity in the N.Acc.

\section{Different Neural Circuits for \\ Foraging Tasks With or Without a Delay}

The finding that DA activity can modulate hippocampal inputs to the N.Acc. when a rat is searching for food in the random foraging task parallels a related study on the role of dopaminergic modulation of vSub inputs to the PFC during foraging on a delayed version of the radialarm maze task (Seamans et al., 1998). Infusions of a $D_{1}$ receptor antagonist into the prelimbic region of the PFC have selectively disrupted foraging during a delayed spatial win-shift task, in which rats were required to use prospective memory to locate food on a radial maze following a delay (Cook, Brown, \& Riley, 1985; Floresco, Seamans, $\&$ Phillips, 1997). However, similar infusions did not disrupt performance of the random foraging task, in which animals would forage retrospectively (Cook et al., 1985; Floresco, Seamans, \& Phillips, 1997). In addition, asymmetric inactivations of the vSub in combination with contralateral infusions of SCH 23390 into the prelimbic cortex also disrupted delayed spatial win-shift performance (Seamans et al., 1998). Collectively, our data suggest that there is a double dissociation between the func- 
tion of brain regions that require DA activity during either delayed or nondelayed foraging. Specifically, $D_{1}$ receptor stimulation in the N.Acc. is essential during exploration when an animal has no previous information about the location of reward in an environment, but is not required when the animal has the benefit of such prior knowledge. Conversely, $D_{1}$ receptor activity is essential in the prelimbic cortex when an animal must use previously acquired information about the probable location of food to forage efficiently, whereas DA activity in the prelimbic cortex is inconsequential when an animal forages with no prior information about the location of food. It is important to note that although separate neural circuits mediate these two different types of foraging behavior, DA modulation of hippocampal inputs forms an essential feature of both circuits.

It is well recognized that the N.Acc. serves as an interface between different cortico-limbic information processing systems and as such plays a critical role in processes that determine response priorities (Mogenson et al., 1993). On the basis of the evidence discussed above, it would appear unlikely that neural activity within the N.Acc. is involved directly in establishing the goals and priorities for the organism. These functions appear to be performed by systems within the temporal and frontal cortices; in contrast, the N.Acc. serves simply as a sophisticated relay system, the output of which is dictated by the biasing of different cortico-limbic afferents in the manner described above. Appropriate response strategies for specific environmental contingencies may be determined by several different processes, including preexisting stimulus-reward associations, such as those formed in the amygdala (Everitt et al., 1991); spatial and contextual information derived from the hippocampus (Floresco, Seamans, \& Phillips, 1997); or prospective memory for a temporal sequence of responding, mediated by the PFC (Floresco, Braaksma, \& Phillips, 1997). The capacity of these different networks to bias the internal functioning of the N.Acc. may well determine which alternative response strategy will gain direct access to the motor system via efferents from the N.Acc. to the ventral pallidum. In this sense, the function of the N.Acc. is to facilitate the transformation of cognitive processes into meaningful patterns of behavior, without being involved in cognition per se.

\section{REFERENCES}

Bardo, M. T., Bowling, S. L., Robinet, P. M., Rowlett, J. K., Lacy, M., \& MAtTingly, B. A. (1993). Role of dopamine $D_{1}$ and $D_{2}$ receptors in novelty-maintained place preference. Experimental \& Clinical Psychopharmacology, 1, 101-109.

Barnes, C. A., MCNaughton, B. L., Mizumori, S. J., Leonard, B. W., \& LiN, L. H. (1990). Comparison of spatial and temporal characteristics of neuronal activity in sequential stages of hippocampal processing. Progress in Brain Research, 83, 287-300.

Berns, G. S., Cohen, J. D., \& Mintun, M. A. (1997, May). Brain regions responsive to novelty in the absence of awareness. Science, 276, 1272-1275.

Blackburn, J. R., Phillips, A. G., \& Fibiger, H. C. (1987). Dopamine and preparatory behavior: I. Effects of pimozide. Behavioral Neuroscience, 101, 352-360.
Blaha, C. D., Yang, C. R., Floresco, S. B., Barr, A. M., \& Phillips, A. G. (1997). Stimulation of the ventral subiculum of the hippocampus evokes glutamate receptor-mediated changes in dopamine efflux in the rat nucleus accumbens. European Journal of Neuroscience, 5 , 902-911.

Brog, J. S., Salyapongse, A., Deutch, A., \& Zahm, D. S. (1993). The pattern of afferent innervation of the core and shell in the "accumbens" part of the ventral striatum: Immunohistochemical detection of retrogradely transported Fluoro-Gold. Journal of Comparative Neurology, 338, 255-278.

BrudzynSKI, S. M., \& Krol, S. (1997). Role of the subiculumaccumbens pathway in the initiation of locomotor exploration. Journal of Psychopharmacology, 11S, A20.

Buhusi, C. A., \& SchmajuK, N. A. (1996). Attention, configuration, and hippocampal function. Hippocampus, 6, 621-642.

Burns, L. H., Annett, L., Kelley, A. E., Everitt, B. J., \& Robbins, T. W. (1996). Effects of lesions to amygdala, ventral subiculum, medial prefrontal cortex, and nucleus accumbens on the reaction to novelty: Implications for limbic-striatal interactions. Behavioral Neuroscience, 110, 60-73.

Cador, M., Robbins, T. W., Everitt, B. J., Simon, H., Le Moal, M., \& STINUS, L. (1991). Limbic-striatal interactions in reward related processes: Modulation by the dopaminergic system. In P. Willner \& J. Scheel-Kruger (Eds.), The mesolimbic dopamine system (pp. 225251). New York: Wiley.

COOK, R. G., BRown, R. F., \& Riley, D. A. (1985). Flexible memory processing by rats: Use of prospective and retrospective information in the radial arm maze. Journal of Experimental Psychology: Animal Behavior Processes, 11, 453-469.

DeFrance, J. F., Sikes, R. W., \& Chronister, R. B. (1985). Dopamine action in the nucleus accumbens. Journal of Neurophysiology, 54, 1568-1577.

Everitt, B. J., Morris, K. A., O’Brien, A., \& Robbins, T. W. (1991). The basolateral amygdala-ventral striatal systems and conditioned place preference: Further evidence of limbic-striatal interactions underlying reward-related processes. Neuroscience, 41, 1-18.

Floresco, S. B., BraAksma, D. N., \& Phillips, A. G. (1997). Interactions between the prefrontal cortex, ventral striatum and medialdorsal thalamus mediate delayed responding on a radial-arm maze task. Society for Neuroscience Abstracts, 23, 1598.

Floresco, S. B., Seamans, J. S., \& Phillips, A. G. (1996). A selective role for dopamine in the nucleus accumbens of the rat in random foraging but not delayed spatial win-shift-based foraging. Behavioural Brain Research, 80, 161-168.

Floresco, S. B., Seamans, J. K., \& Phillips, A. G. (1997). Selective roles for hippocampal, prefrontal cortical, and ventral striatal circuits in radial-arm maze tasks with or without a delay. Journal of Neuroscience, 17, 1880-1890.

Fuller, T. A., Russchen, F. T., \& PRICE, J. L. (1987). Sources of presumptive glutamatergic/aspartergic afferents to the rat ventral striatopallidal region. Journal of Comparative Neurology, 258, 317-338.

Gonon, F., \& Sundstrom, L. (1996). Excitatory effects of dopamine released by impulse flow in the rat nucleus accumbens in vivo. Neuroscience, 75, 13-18.

GracY, K. N., \& PICKel, V. M. (1996). Ultrastructural immunocytochemical localization of the $N$-methyl-D-aspartate receptor and tyrosine hydroxylase in the shell of the rat nucleus accumbens. Brain Research, 739, 169-181.

Groenewegen, H. J., Vermeulen-Van der Zee, E., te Kortschot, A., \& WITTER, M. P. (1987). Organization of the projections from the subiculum to the ventral striatum in the rat: A study using anterograde transport of Phaseolus vulgaris leucoagglutinin. Neuroscience, 23, 103-120.

HaRvey, J., \& LACEY, M. G. (1996). Endogenous and exogenous dopamine depress EPSCs in rat nucleus accumbens in vitro via $D_{1}$ receptor activation. Journal of Physiology, 492, 143-154.

Hiroi, N., \& White, N. M. (1990). The reserpine-sensitive dopamine pool mediates $(+)$-amphetamine-conditioned reward in the place preference paradigm. Brain Research, 510, 33-42.

Hooks, M. S., \& Kalivas, P. W. (1995). The role of mesoaccumbenspallidal circuitry in novelty-induced behavioral activation. Neuroscience, 64, 587-597. 
Hu, X. T., \& WANG, R. Y. (1988). Comparison of effects of D-1 and D-2 dopamine receptor agonists on neurons in the rat caudate putamen: An electrophysiological study. Journal of Neuroscience, $\mathbf{8}$, 4340-4348.

KIM, J. S., \& LEviN, E. D. (1996). Nicotinic, muscarinic and dopaminergic actions in the ventral hippocampus and the nucleus accumbens: Effects on spatial working memory in rats. Brain Research, 725, 231240.

KiYATKin, E. A., \& Rebec, G. V. (1996). Dopaminergic modulation of glutamate-induced excitations of neurons in the neostriatum and nucleus accumbens of awake, unrestrained rats. Journal of Neurophysiology, 75, 142-153.

LAvoIE, A. M., \& Mizumori, S. J. (1994). Spatial, movement- and reward-sensitive discharge by medial ventral striatum neurons in rats. Brain Research, 638, 157-168.

McDonald, R. J., \& White, N. M. (1993). A triple dissociation of memory systems: Hippocampus, amygdala, and dorsal striatum. $B e-$ havioral Neuroscience, 107, 3-22.

Mogenson, G. J., Brudzynski, S. M., Wu, M., YANG, C. R., \& Yim, C. Y. (1993). From motivation to action: A review of dopaminergic regulation of limbic $\rightarrow$ nucleus accumbens $\rightarrow$ ventral pallidum $\rightarrow$ pedunculopontine nucleus circuitries involved with limbic-motor integration. In P. W. Kalivas \& C. D. Barnes (Eds.), Limbic-motor circuits and neuropsychiatry (pp. 193-263). Boca Raton, FL: CRC Press.

Mogenson, G. J., \& NiELSEN, M. (1984). A study of the contribution of hippocampal-accumbens-subpallidal projections to locomotor activity. Behavioral \& Neural Biology, 42, 38-51.

Muller, R. (1996). A quarter of a century of place cells. Neuron, 17, 813-822.

O'KeEFE, J., \& NADEL, L. (1978). The hippocampus as a cognitive map. Oxford: Oxford University Press

Olton, D. S., \& PAPAS, B. C. (1979). Spatial memory and hippocampal function. Neuropsychologia, 17, 669-682.

PaXinos, G., \& WaTson, C. (1997). The rat brain in stereotaxic coordinates (3rd ed.). San Diego: Academic Press.

Pennartz, C. M. A., Dolleman-vender Weel, M. J., Kitai, S. T., \& Lopes DA SilvA, F. H. (1991). Presynaptic dopamine $D_{1}$ receptors attenuate excitatory and inhibitory inputs to the shell region of the rat nucleus accumbens studied in vitro. Journal of Neurophysiology, 67, $1325-1333$.

Pennartz, C. M. A., Groenewegen, H. J., \& Lopes Da Silva, F. H. (1994). The nucleus accumbens as a complex of functionally distinct neuronal ensembles: An integration of behavioural, electrophysiological and anatomical data. Progress in Neurobiology, 42, 719-761.

Ploeger, G. E., Spruijt, B. M., \& Cools, A. R. (1994). Spatial localization in the Morris water maze in rats: Acquisition is affected by intra-accumbens injections of the dopaminergic antagonist haloperidol. Behavioral Neuroscience, 108, 927-934.

RobBins, T. W., \& EveritT, B. J. (1992). Functions of dopamine in the dorsal and ventral striatum. Seminars in the Neurosciences, 4, 119-127.

SALAmone, J. D., Cousins, M. S., \& SNYder, B. J. (1997). Behavioral functions of nucleus accumbens dopamine: Empirical and conceptual problems with the anhedonia hypothesis. Neuroscience \& Biobehavioral Reviews, 21, 341-359.

Save, E., Poucet, B., Foreman, N., \& Buhot, M.-C. (1992). Object exploration and reactions to spatial and nonspatial changes in hooded rats following damage to parietal cortex or hippocampal formation. Behavioral Neuroscience, 106, 447-456.

ScHULTZ, W. (1997). Dopamine neurons and their role in reward mechanisms. Current Opinion in Neurobiology, 7, 191-197.

Seamans, J. K., Floresco, S. B., \& Phillips, A. G. (1998). D 1 receptor modulation of hippocampal-prefrontal cortical circuits integrating spatial memory with executive functions in the rat. Journal of Neuroscience, 18, 1613-1621.

SesaCK, S. R., \& PICKEL, V. M. (1990). In the rat medial nucleus accumbens, hippocampal and catecholaminergic terminals converge on spiny neurons and are in apposition to each other. Brain Research, 527, 266-279.

Spyraki, C., Fibiger, H. C., \& Phillips, A. G. (1982a). Attenuation by haloperidol of place preference conditioning using food reinforcement. Psychopharmacology, 77, 379-382.

Spyraki, C., Fibiger, H. C., \& Phillips, A. G. (1982b). Dopaminergic substrates of amphetamine-induced place preference conditioning Brain Research, 253, 185-193.

TOTTERdell, S., \& SMith, A. D. (1989). Convergence of hippocampal and dopaminergic input onto identified neurons in the nucleus accumbens of the rat. Journal of Chemical Neuroanatomy, 2, 285-298.

Wolterink, G., Phillips, G., Cador, M., DonselaAr-Wolterink, I., RobBins, T. W., \& EveritT, B. J. (1993). Relative roles of ventral striatal $\mathrm{D}_{1}$ and $\mathrm{D}_{2}$ dopamine receptors in responding with conditioned reinforcement. Psychopharmacology, 110, 355-364.

WU, M., \& BRUDZYNSKI, S. M. (1995). Mesolimbic dopamine terminals and locomotor activity induced from the subiculum. NeuroReport, $\mathbf{6}$, 1601-1604.

Wu, M., Brudzynski, S. M., \& Mogenson, G. J. (1993). Differential effects of quinpirole in the nucleus accumbens depending on the initial level of locomotor activity. Brain Research Bulletin, 32, 395-398.

YANG, C. R., \& Mogenson, G. J. (1984). Electrophysiological responses of neurones in the nucleus accumbens to hippocampal stimulation and the attenuation of the excitatory responses by the mesolimbic dopaminergic system. Brain Research, 324, 69-84.

YANG, C. R., \& Mogenson, G. J. (1986). Dopamine enhances terminal excitability of hippocampal-accumbens neurons via $D_{2}$ receptor: Role of dopamine in presynaptic inhibition. Journal of Neuroscience, 6, 2470-2478.

YANG, C. R., \& Mogenson, G. J. (1987). Hippocampal signal transmission to the mesencephalic locomotor regions and its regulation by dopamine D-2 receptors in the nucleus accumbens: An electrophysiological and behavioral study. Neuroscience, 23, 1041-1055.

(Manuscript received July 3, 1998;

revision accepted for publication December 21,1998 .) 